\title{
Ideas de los estudiantes acerca de la ciencia, los científicos y las carreras de ciencia
}

VICTORIA EUGENIA GUTIÉRREZ

Departamento de Educacion/UAA

\section{INTRODUCCIÓN}

$\mathbb{E}$

n este artículo se presentan algunos de los resultados obtenidos en un estudio realizado en la Universidad Autónoma de Aguascalientes con 407 jóvenes que en el semestre enero-junio de 1995 cursaban sus estudios en 20 licenciaturas. Uno de los objetivos de este trabajo fue el conocer las actitudes que los estudiantes universitarios manifiestan hacia la ciencia, hacia los científicos y hacia las llamadas carreras de ciencia. Para el logro de este propósito fue diseñado un cuestionario que exploraba las tres dimensiones que el modelo teórico tradicional de las actitudes propone, esto es: el componente cognitivo, el componente afectivo y el componente connativo o de tendencia a la acción. ${ }^{1}$

Los estudios sobre aspectos afectivos en la enseñanza y el aprendizaje de la ciencia han empezado a recibir especial atención. Según T. Koballa, la importancia de las variables afectivas en los procesos de enseñar y aprender empieza a ser reconocida. ${ }^{2} \mathrm{El}$

1. Anderson, L. "Attitudes and their measurement", in T. Husen y Postlethwaite (eds.) The International Encyclopedia of Education Reasearch and Studies, Oxford, Pergamon Press, 1988, pp. 5318-5324.

2. "Attitude and related concepts in Science education". Science education, Vol.3, (6), 1988, pp. 45-56. 
rendimiento de los estudiantes se ve influido no sólo por las variables cognitivas sino por un conjunto de factores que tienen que ver con la dimensión afectiva de las personas.

Dentro de este tipo de estudios, los referidos a las actitudes hacia la ciencia constituyen una de las temáticas más abordadas. Puede decirse que es un asunto siempre presente, cuando se habla de educación científica. Son indicadores de este interés: el número de informes sobre investigaciones que se ocupan del estudio de las actitudes; la cantidad de técnicas reportadas para medir estas variables, el considerable número de referencias sobre el particular, encontradas tanto en fuentes primarias como secundarias, etc. De hecho, las actitudes hacia la ciencia ocupan un lugar importante en la literatura sobre el tema. D. Hall sostiene que en un estudio reciente sobre prioridades de investigación, son las actitudes el interés principal de la educación científica. ${ }^{3}$

El trabajo que se presenta puede ser ubicado en el marco de la llamada Educación científica, o como otros autores la llaman la Enseñanza y el aprendizaje de la ciencia.

\section{La educación científica y sus fines}

Tradicionalmente, se ha considerado que la educación científica hace referencia únicamente a la adquisición de un conjunto de conocimientos en un campo disciplinar específico; o bien, en muchos de los casos, a una forma de enseñar ciencia en la que son utilizados recursos didácticos sofisticados. Sin embargo, las nociones anteriores sobre este tipo de educación, están siendo superadas. Actualmente se propone una concepción en términos más críticos. Se trata ahora de una formación más compleja en la que el individuo deberá

3. "The influence of an innovative activity-centered biology program on attitudes toward science teaching among preservice elementary teachers". In Science Education, Vol. 92, 5, 1992, pp. 239-242. 
poseer-además de los conocimientos específicos sobre determinado campo disciplinar- dominio de los métodos y las técnicas empleadas en el trabajo científico; conocimiento sobre los fundamentos de la ciencia y actitudes críticas ante este tipo particular de saber.

En este sentido, la educación científica pretende formar una disposición favorable hacia la actividad científica y los científicos, y en algunos casos, llevar esa buena disposición hasta el deseo de ser parte dinámica de dicha actividad. Lo anterior puede lograrse si en la educación científica son atendidos tanto los aspectos cognitivos como la dimensión afectiva, particularmente la que se refiere a las actitudes y los valores. Este es el reto de educar en la ciencia y para la ciencia.

En el terreno de la educación científica son planteados tres tipos de fines, que se pretende orienten un proyecto educativo y sean alcanzados en todos los niveles de escolaridad. Los llamados fines formativos plantean que el alumno deberá desarrollar capacidades básicas que le permitan la formación de habilidades y actitudes. Los fines instructivos aspiran a que el alumno domine conocimientos básicos acerca de la ciencia; los llamados fines utilitarios señalan como meta, que una vez dominado el conocimiento, el alumno tenga un mejor desenvolvimiento en su medio social.

\section{Los problemas que enfrenta la educación científica}

Si bien se han reconocido aciertos en la enseñanza y el aprendizaje de la ciencia, son innegables los numerosos obstáculos que impiden la consecución plena de los fines de la educación científica.

Algunos de los problemas identificados tienen que ver con la pobre comprensión que tienen los estudiantes sobre la naturaleza del trabajo científico y de los métodos a través de los cuales el conocimiento científico es construido, o con la incapacidad de muchos profesores, incluso los experimentados, para comunicar significados de conceptos y principios básicos. Otros problemas surgen por el vacío en la mayoría de los sistemas educativos respecto a incor- 
porar las perspectivas epistemológicas modernas, lo cual, sin duda, incide en las concepciones que los estudiantes tienen sobre la ciencia. Se ha encontrado también que un buen número de problemas surgen por el poco interés o la indisposición que manifiestan los estudiantes hacia los contenidos, las materias, los profesores y las carreras de ciencia. Tales indisposiciones se traducen en bajo rendimiento de los estudiantes, fracaso escolar, descenso en la matrícula de carreras científicas, etc. Todos estos problemas se convierten en asunto de interés para los investigadores educativos generándose así todo un campo de investigación.

\section{La investigación sobre la enseñanza y el aprendizaje de la ciencia}

La educación científica además de objeto de la reflexión pedagógica es preocupación de la investigación educativa. Numerosos trabajos cuyo foco de estudio es tanto la enseñanza, como el aprendizaje de la ciencia, aparecen cada vez con mayor frecuencia en la literatura sobre educación. Algunos autores, en un intento de organización de lo hecho en este campo, presentan las líneas de investigación que se han seguido en los distintos estudios. Tal es el caso de Moreira y Novak, quienes clasifican los trabajos realizados bajo las siguientes perspectivas: el alumno, el profesor, la materia de enseñanza, el medio social y la evaluación. ${ }^{4}$

Los hallazgos que a continuación se presentan son resultado $0^{5}$ -como se señaló al principio de este documento- de un estudio bajo

4. "Investigación en la enseñanza de las ciencias en la Universidad de Cornell: esquemas teóricos, cuestiones centrales y abordes metodológicos", Enseñanza de las ciencias, Vol. 6 (1), 1988, pp. 3-18.

5. Los resultados se refieren únicamente a la exploración del componente cognitivo de la actitud en sus tres tipos de creencias: descriptivas, evaluativas y recomendativas. La información sobre los otros dos componentes de la actitud -léase componente afectivo y de tendencia a la acción- así como los resultados de las variables independientes y la relación entre éstas y las actitudes de los estudiantes, se encuentra en el informe de esta investigación. 
la línea de investigación que pretende explorar las concepciones y opiniones de los estudiantes acerca de la ciencia, el conocimiento cientifico y los científicos, así como el conocimiento de variables afectivas consideradas como elementos determinantes que actúan en el proceso de enseñar y aprender ciencia.

\section{Creencias descriptivas sobre la ciencia}

Una de las aportaciones de este estudio es que han sido medidos, de manera separada, cada uno de los componentes que estructuran la actitud, a diferencia de un buen número de trabajos en los que se atiende uno solo de los componentes -casi siempre el afectivo- y se infieren los otros dos. Además de medir los tres componentes ya citados, en el componente cognitivo han sido explorados los distintos tipos de creencias que lo conforman: creencias descriptivas, creencias evaluativas y creencias recomendativas.

Para la medición de las llamadas creencias descriptivas o información que la persona tiene sobre la ciencia, se presentaron varias escalas en las que se pedía al estudiante identificar la naturaleza de distintas actividades, programas y revistas de divulgación de la ciencia, científicos y carreras de ciencia. Al respecto se observó:

De 5 actividades de tipo científico, los estudiantes califican sólo a 2, como de tal naturaleza. Parece que no hay problema en señalar que la búsqueda de una vacuna $(81 \%)$ o el hacer experimentos (64\%) son actividades científicas. Sin embargo, estudios del campo de las ciencias sociales o del hombre, no se consideran de carácter científico. Ante trabajos de este tipo los estudiantes opinaron que se trataba de actividades 0 tareas muy específicas independientes de la investigación. Es el caso, por ejemplo, de la siguiente actividad: realizar un estudio para determinar la relación entre conductas agresivas de los niños y el tiempo dedicado a ver programas de violencia por televisión : sólo el $27 \%$ opina que se trata de un estudio científico. Lo mismo sucede con otras actividades similares: 
comparar el tipo de problemas de aprendizaje que predominan en zonas rurales y zonas urbanas y realizar un estudio en el que se determinen los índices de criminalidad y los tipos de homicidio por zona geográfica, sólo un $20 \%$ y $14.5 \%$ respectivamente señalaron que podrían ser consideradas como investigaciones.

Otro tipo de tareas, que si bien pueden formar parte de un estudio, pero que no constituyen en sí mismas un trabajo de naturaleza científica, son percibidas por los estudiantes como actividades de investigación. Tales tareas son por ejemplo: tomar muestras para el análisis de ciertas sustancias (54.5\% la califica como actividad científica); hacer cultivos en el laboratorio (52.1\% la considera como ejemplo o modalidad de investigación científica). Lo anterior sugiere que actividades que hacen referencia a trabajo de laboratorio y que se perciben como de cierta complejidad, se asocian con hacer ciencia.

De un conjunto de actividades profesionales que se fundamentan en la ciencia, los estudiantes opinan que acciones como: representar la parte acusadora ante un tribunal competente en un fraude, es una actividad ajena a la ciencia (70\%). Opinión semejante es emitida acerca de brindar asesoría a una escuela de padres de familia con hijos adolescentes, el 64\% la considera como ajena a la ciencia. La acción de poner en marcha el desarrollo de un programa comunitario es considerada por el $70 \%$ como una tarea que es ajena totalmente a la ciencia. Caben las preguntas: ¿por qué se percibe la actividad profesional como algo ajeno a la ciencia? ¿Se considera que la formación para una profesión no tiene fundamento científico? En cambio, en el caso de prescribir un tratamiento a un paciente diabético con base en un análisis de sangre y un diagnóstico clínico completo, casi el $50 \%$ opina que se trata de una actividad científica. En el análisis sobre las carreras que los estudiantes identifican -y que es presentado más adelante- como científicas u orientadas hacia la ciencia se aprecia que la carrera de Medicina es percibida como de esta naturaleza. 
Sobre actividades ajenas a la ciencia los estudiantes no presentaron problemas. Claramente identificaron aquellos planteamientos que hacían referencia a actividades que en ningún caso pueden formar parte de la investigación científica.

$\mathrm{Al}$ analizar esta información con la lógica de un examen en el que se asignen puntajes a respuestas correctas e incorrectas, y se brinde además una calificación (con los criterios tradicionales) se encuentra que el 83\% (338 estudiantes de una muestra de 407) no identifica correctamente la naturaleza de las distintas actividades. Si creamos dos grupos -basándonos en la información anterior- de estudiantes aprobados y no aprobados, el porcentaje mayor de estudiantes se ubicaría entre los no aprobados. En términos de promedio del grupo se hablaría de un puntaje alcanzado igual a 4.3.

Por lo que se refiere a la identificación de revistas de divulgación científica, se encuentra que un porcentaje alto de estudiantes (70\%) reconoce títulos de este tipo de publicaciones (obviamente los títulos de las revistas, en muchos casos, son muy sugerentes de su contenido y esto pudiera facilitar su identificación). Las dificultades empiezan cuando hay que discriminar entre aquellas revistas que no son de corte científico. En este tipo de publicaciones aumentó el porcentaje en la categoría "no sé". Cuando se solicitó a los alumnos que señalaran el título de otra revista de divulgación científica, distinta a las que aparecían en el listado, el 50.5\% expresó no conocer otra; sólo $29.5 \%$ contestó a la pregunta. De este porcentaje, la mayoría contestó de manera equivocada citando las siguientes revistas: PC Tips, Proceso, Nexos, PC Magacine, PC computadoras, Geomundo, Selecciones del Reader's Digest, Año Cero, Life Time, Sabías tú qué, Época, Contenido, Casa y Gente, Automundo y Mecánica Popular. Únicamente el $12.5 \%$ aportó títulos de revistas de divulgación científica, entre las que se encuentran: American Science, Medical Practice, MSD, Revista del Centro de Estudios Educativos, Behavior Journal y Perfiles Educativos.

Si esta información es tratada como la referente a actividades científicas (esto es con la lógica de un examen, en el que se asignen 
puntajes a respuestas correctas e incorrectas y se asignen calificaciones) se tiene que a diferencia de la identificación de actividades de naturaleza científica, el grupo mayor es el de los aprobados $(60 \%)$. En términos de promedio del grupo (total de la muestra) la calificación es de 6 .

En cuanto a la identificación de programas de divulgación científica, se observa que en general, los estudiantes conocen e identifican este tipo de programas (casi un 70\%). A la demanda de que agregaran el nombre de un programa de este tipo, la mitad de la muestra de alumnos expresó no conocer otro distinto a los ya enlistados. De los que aportaron el nombre de un programa (100 jóvenes), la mitad responde de manera incorrecta señalando emisiones como: Para gente grande, Nuestra arquitectura, Aunque usted no lo crea y Super ondas. Los estudiantes que contestaron de manera correcta (50 alumnos) consideran programas de divulgación del quehacer científico: El universo, El cuerpo humano, Mundo animal, La naturaleza, Especiales de Carl Sagan, Grandes inventos, Científicos e inventores; algunos estudiantes señalan: "toda la programación del Discovery Chanel". Efectivamente, casi todos los programas citados por los estudiantes que contestan correctamente a esta pregunta, corresponden a la programación de este canal (transmitido por cable). Si la información se presenta como se ha venido haciendo, se tiene que el grupo mayor es el de los aprobados (66\%). El promedio resultante es de 6.3 .

\section{Creencias descriptivas sobre carreras científicas}

Ante un listado de 20 carreras (científicas y no científicas) los estudiantes identificaron sin problema carreras de ciencia de las áreas duras, no así carreras de ciencia en las áreas sociales o carreras con perfiles no muy conocidos (Urbanismo y Economía, por ejemplo), las cuales consideraron como no científicas. El único caso que escapa es el de la Licenciatura en Educación Especialidad Investi- 
gación Educativa, la cual es percibida, por la mayoría de los sujetos de la muestra, como carrera científica $(66 \%)$. El promedio resultante es de 6.3.

En cuanto a las carreras no científicas, fueron fácilmente identificables aquéllas con perfiles muy conocidos (Contador público, Derecho, Administración de Empresas, Arquitectura, etc.) En el caso de carreras como Ingeniería en Sistemas (que fue calificada como carrera de ciencia) es posible que se perciba como de cierta complejidad y muy asociada a la tecnología de punta, de ahí que merezca este adjetivo.

La carrera de Medicina es considerada por un 89\% de los estudiantes como una carrera científica. Tal vez su situación de prestigio, de definición de su campo, el percibirla como de cierta complejidad por el objeto de estudio del que se ocupa, etc., motive a que los estudiantes la identifiquen así.

En términos de puntajes obtenidos puede decirse que el $54.3 \%$ de los alumnos no identificó de manera correcta entre las carreras científicas y no científicas. El promedio alcanzado fue de 5.4.

Además de determinar si los estudiantes identificaban carreras orientadas a la producción de conocimiento, se intentó explorar su percepción sobre algunas dimensiones como: la facilidad o dificultad para conseguir trabajo; la percepción de ingresos altos-bajos; la necesidad de tener una gran-poca capacidad; el futuro (carreras con gran futuro o poco futuro), etc., tanto de carreras científicas como no científicas. Fueron presentadas tres carreras científicas (Matemáticas Aplicadas, Biología y Sociología) y tres no científicas (Medicina, Ingeniería Civil y Derecho). Los estudiantes señalaron que existe mayor dificultad para conseguir empleo en carreras de corte científico; que los ingresos en dichas carreras son bajos, tienen poco futuro y bajo prestigio. En cuanto a la capacidad requerida para desempeñar actividades profesionales, podría decirse que los estudiantes no manifiestan mucha diferencia: Matemáticas Aplicadas y Medicina son consideradas como carreras que demandan mucha 
capacidad (se trata de una carrera científica y otra de corte no orientado hacia la ciencia). Llama la atención que la carrera de Sociología sea percibida como la de más baja demanda en términos de capacidad. Se confirma que la carrera de Medicina es percibida por los estudiantes como de altos ingresos, gran capacidad, mucho futuro y alto prestigio.

\section{Creencias descriptivas sobre científicos}

En esta escala fueron presentados 14 personajes. De los 7 científicos que se enlistaron, el 90\% de los estudiantes identificó sin dificultad a 4 científicos clásicos, de renombre (Einstein, Pasteur, Newton y Darwin) todos procedentes de las ciencias duras. Sólo un científico del área de ciencias del hombre fue reconocido por un $33 \%$ de los alumnos, lo mismo sucedió con un científico contemporáneo de las ciencias duras (Stephen Hawking). Los estudiantes identificaron también personajes que claramente no son científicos (Juan Pablo II y Pablo Picasso). Sin embargo, señalan no saber si Uri Geller, Rodolfo Benavides u Orus Solimar son científicos o no. A la solicitud de que se aportara el nombre de un científico, un 67\% (270 estudiantes) contestó tal demanda; de dicho porcentaje, la mitad (135) señaló los siguientes nombres de científicos: Niels Henrik Bohr, Galileo Galilei, Alejandro Volta, Nicolás Copérnico, Juan Gregorio Mendel, Antonio Lavoisier, Pedro y Marie Curie, Roberto Koch, Leonardo De Vinci, Celsius, Blas Pascal, Henry Wallon, Juan Pavlov, Torricelli, Tomás Alva Edison, Sigmund Freud, Jonás Salk y Roberto Hooke. Sólo 53 estudiantes (de los que contestaron a la pregunta) suponen -de manera incorrecta- que son científicos: Julio Verne, Jaime Mausán, Carlos Fuentes y Octavio Paz. De la identificación de científicos el grupo ligeramente más alto (54\%) fue el de los aprobados y el promedio grupal de 6.3.

Recapitulación: el componente cognitivo en el que se exploraron las creencias descriptivas de los estudiantes sobre la ciencia, carre- 
ras científicas y científicos, puede observarse a través de los resultados obtenidos en términos de porcentajes de aprobación en cada una de las subescalas medidas y mediante el promedio que obtuvo el grupo en las mismas subescalas. La siguiente tabla presenta la información.

Componente cognitivo creencias descriptivas

\begin{tabular}{|c|c|c|}
\hline SUBESCALA & $\begin{array}{c}\text { PORCENTAJE } \\
\text { DE APROBACION }\end{array}$ & $\begin{array}{c}\text { PROMEDIO } \\
\text { DEL GRUPO }\end{array}$ \\
\hline Ciencia & & 4.3 \\
Actividades cientificas & $17.0^{(69)^{*}}$ & 6.0 \\
Revistas científicas & $60.0^{(2+3)}$ & 6.3 \\
Programas científicos & $66.1^{(269)}$ & 5.4 \\
Carreras de ciencia & $46.0^{(186)}$ & 6.3 \\
\hline Científicos & $54.0^{(218)}$ & \\
\hline
\end{tabular}

La primera columna muestra bajos porcentajes de estudiantes que identifican cuestiones de ciencia. En la subescala de ciencia se aprecia que se identifican más programas y revistas científicas que actividades de ciencia. En cuanto a las subescalas de científicos y carreras, es la de científicos en la que se concentra el porcentaje mayor. La segunda columna indica el promedio que el conjunto de los estudiantes obtuvo en cada una de las subescalas, se trata de promedios en general bajos, dos de ellos reprobatorios. De manera más sintética se puede señalar que el porcentaje más alto de

* Corresponde a las frecuencias

$$
\begin{array}{lllllllllllllll}
C & \text { A } & \text { L } & \text { E } & \text { I } & \text { D } & O & \text { S } & C & \text { O } & \text { P } & \text { I } & \text { O } & 83
\end{array}
$$


estudiantes de esta muestra, no identifica de manera correcta actividades de ciencia; programas y revistas de divulgación científica; carreras científicas y científicos.

En términos de actitudes se dice que el componente cognitivo se manifiesta a través de un conocimiento que va de lo mínimo a lo exhaustivo sobre el objeto de la actitud; en este caso puede señalarse que los estudiantes manifiestan un conocimiento mínimo acerca de la ciencia.

\section{Creencias evaluativo/recomendativas sobre la ciencia}

En el componente cognitivo se midieron también las llamadas creencias evaluativo/recomendativas acerca de la ciencia, los científicos y su actividad. El $76 \%$ de los estudiantes manifestó una percepción matizada, moderada sobre el valor de la ciencia. Ante planteamientos muy optimistas, casi ingenuos sobre la utilidad de la actividad científica, emitieron evaluaciones críticas. Lo mismo sucedió con formulaciones muy pesimistas o desfavorables sobre la ciencia. En cambio a enunciados que postulaban visiones de esta empresa en la que se reconocen tanto sus aportaciones como sus efectos secundarios, los alumnos emitieron un mayor grado de acuerdo. Los datos permiten afirmar que en general, si bien se muestra una tendencia favorable, positiva hacia la ciencia, también se manifiesta una visión objetiva acerca de su valor.

\section{Creencias evaluativas sobre los científicos}

El porcentaje más alto (70\%) correponde al acuerdo de los estudiantes con características favorables -aunque estereotipadas- acerca de los científicos. Con los enunciados desfavorables, se aprecia un desacuerdo matizado y se observa que aumentan los porcentajes en zonas de neutralidad. Si atendemos únicamente a los porcentajes más altos e intentamos caracterizar a los científicos, según la muestra 
estudiada, podemos señalar que son percibidos como buscadores de la verdad, muy críticos, con mentes privilegiadas, bien intencionados y amorales.

\section{Creencias evaluativas sobre el trabajo que realizan los científicos}

De acuerdo con los estudiantes (y atendiendo a los porcentajes mayores de cada uno de los atributos considerados, casi siempre arriba del 80\%) el trabajo de los científicos es percibido como útil, bueno, profundo, estimulante, apasionante, dinámico, agradable, benéfico, divertido, sistemático, prestigioso y crítico. Con esta información se puede sostener que hay una evaluación favorable acerca de la actividad que realizan los científicos. La consideración de adjetivos como complejo, difícil y extraño (características que tuvieron también altos porcentajes, por encima del 70\%), muestran, por otro lado, la imagen esterotipada de la actividad científica.

\section{Conclusiones}

Distintos autores ${ }^{6}$ sostienen que el componente cognitivo de la actitud consiste en un conjunto de categorías que los seres humanos utilizan para dar nombre a todos los estímulos. Este componente abarca todo tipo de creencias acerca del objeto de la actitud. Dichas creencias son de naturaleza variada: creencias descriptivas, creencias evaluativas y creencias recomendativas.

Las creencias descriptivas se refieren a la información que tiene el sujeto sobre el objeto en cuestión. Se dice también que la representación cognitiva de un objeto, esto es, el conocimiento que se tenga sobre el objeto no necesariamente es muy exacto. A menu-

6. Gerardo Marín, Manual de investigación en psicología social. México, Trillas, Cap. 4, 1977; Aroldo Rodríguez, Psicología. México, Trillas, Caps. 12, 13 y 14, 1987; Walter Scharml, Introducción a la psicología del desarrollo, Barcelona, Herder, 1977. 
do, incluso, la información puede estar distorsionada. Se señala además que el número de elementos que conforman este componente es variable en las personas. Evidentemente, hay objetos acerca de los cuales los sujetos pueden no saber absolutamente nada. Debe observarse también que algunos objetos son más complejos que otros, y por consiguiente, pueden generar mayor o menor información.

Las creencias evaluativas hacen referencia a las cualidades deseables o indeseables, aceptables 0 inaceptables, buenas o malas y verdaderas o falsas. Se consideran también, como formando parte de este componente, aunque no siempre, las creencias acerca de cómo tratar al objeto de la actitud o creencias recomendativas.

La información anterior permitió fundamentar la exploración del componente cognitivo de las actitudes de los estudiantes hacia la ciencia. De ahí que fueran exploradas las diferentes creencias de los sujetos acerca de la ciencia y cuestiones relacionadas con ésta (carreras de ciencia, científicos y su trabajo).

En el terreno de las creencias descriptivas se encontró que los estudiantes de la muestra poseen poca información, en cuanto a la naturaleza 0 características de la actividad científica; incluso puede decirse que dicha información no es del todo exacta. Es decir, hay creencias erróneas acerca de la producción del conocimiento científico. El problema que supone que los estudiantes posean concepciones erróneas sobre la actividad científica es central en la línea de investigación en la enseñanza y aprendizaje de la ciencia. Carrascosa y Fernández ${ }^{i}$ señalan que estudios recientes muestran que sigue transmitiéndose en la escuela una visión muy deformada del trabajo científico.

Han sido ya identificadas algunas visiones erróneas acerca de la actividad científica que la escuela transmite a sus estudiantes. Revisar algunas de ellas permite tener categorías para ubicar las creencias que los estudiantes tienen sobre la naturaleza del trabajo científico. 
Según los autores citados, la escuela enseña una visión empirista y ateórica, en donde se resalta el papel de la observación y la experimentación neutras, no contaminadas por ideas apriorísticas. Se olvida el papel esencial de las hipótesis y la construcción de cuerpos coherentes de conocimientos. El trabajo científico es presentado como una cuestión de descubrimiento.

$\mathrm{Al}$ respecto, en este estudio los alumnos consideraron únicamente como científicas aquellas actividades que claramente estaban relacionadas con procedimientos experimentales, incluso, actividades que pueden realizarse en una investigación, que en sentido estricto no son la investigación pero que hacen alusión a trabajo de laboratorio fueron calificadas como ejemplos de trabajo científico.

Otra visión transmitida acerca de la ciencia es la que la presenta con una visión rígida (algorítmica, exacta, infalible). Aquí el "método científico" es concebido como un conjunto de etapas a seguir mecánicamente. Se resalta el trabajo cuantitativo, control riguroso, objetos de estudio directamente observables; se descarta, e incluso se rechaza todo aquello que significa creatividad, inventiva, duda, etc.

Los estudiantes de la muestra de nuestro estudio calificaron como no científicos, trabajos cuyo objeto de estudio no es directamente observable; es el caso de los estudios en las ciencias del hombre. Tal vez los estudiantes perciban que este tipo de trabajos carecen de rigor. Un trabajo que encuentra resultados similares en este punto es el de Herrera y Barnett, éstos señalan que los estudiantes que entrevistaron consideraron como ciencias, únicamente a las disciplinas duras. ${ }^{8}$

Una visión velada y elitista de la ciencia, es otra de las concepciones transmitida por la escuela. Aquí se dice, se utiliza la com-

7. "Análisis de algunas visiones deformadas sobre la naturaleza de la ciencia y las características del conocimiento científico", Enseñanza de las ciencias. Número extra, 1993, pp. 29-30.

8. "Actitud hacia la ciencia y la enseñanza de la ciencia". Enseñanza de las ciencias. Número extra, 1993, pp. 27-28. 
plejidad del aparato matemático, para mostrar la accesibilidad de la ciencia sólo a unos cuantos. El trabajo científico es presentado como un dominio reservado a las minorías particularmente dotadas, transmitiendo expectativas negativas hacia la mayoría de los alumnos, con claras distinciones de naturaleza, social, sexual (el quehacer científico es visto como actividad eminentemente masculina).

En nuestro trabajo, cuando se cuestionó a los alumnos sobre los científicos y el trabajo que éstos realizan, manifiestaron ideas estereotipadas hacia ambos objetos: los científicos son los buscadores de la verdad, son muy críticos, y tienen mentes privilegiadas. El trabajo que ellos realizan fue valorado de manera muy favorable. Es importante, interesante, valioso, útil, bueno, pero por otro lado es percibido como complejo, difícil y extraño. En el estudio de Acevedo (1993) hay coincidencia con el resultado anterior; el autor expresa que la opinión sobre el trabajo científico fue mucho más favorable de lo esperado, recibiendo en conjunto una valoración muy positiva. ${ }^{9}$

La descontextualización de la ciencia es otra de las visiones transmitidas. La ciencia es presentada como socialmente neutra, los científicos son vistos como seres por encima del bien y el mal, encerrados en torres de marfil y ajenos a las necesarias tomas de decisión. Se cae en una visión simplista de la ciencia (exaltación de la ciencia como factor absoluto de progreso o rechazo sistemático a causa de su capacidad destructiva, efectos contaminantes, etc.).

$\mathrm{Al}$ respecto, los estudiantes que participaron en este estudio muestran una visión un tanto más matizada, más racional del alcance y efectos secundarios de la ciencia. Se esperaba que hubiese una tendencia a concebir la ciencia de manera optimista, ingenua, demasiado confiada en el poder de la ciencia. Sin embargo, no sucedió así.

9. "Actitudes hacia el aprendizaje de las ciencias físicas, naturales y matemáticas en BUP y COU. Un estudio sobre 3 dimensiones". Enseñanza de las ciencias, Número extra, 1993,pp. 13-14. 
Otras visiones transmitidas tienen que ver con el individualismo en el trabajo científico: el conocimiento es obra de genios aislados, se ignora el trabajo colectivo; la visión aproblemática y ahistórica de la ciencia: no se muestran los problemas en su construcción, su evolución, las limitaciones del conocimiento actual o las perspectivas abiertas; la visión exclusivamente analítica: la parcialización simplista del conocimiento que olvida los esfuerzos posteriores de unificación y construcción de cuerpos coherentes de conocimientos cada vez más amplios, de tratamiento de problemas de frontera entre distintos cuerpos de conocimientos que pueden llegar a unirse, etc.; la visión acumulativa lineal: los conocimientos aparecen como fruto de crecimiento lineal, ignorando las crisis, las rupturas profundas. Se ignora, en particular, la discontinuidad radical entre el tratamiento científico de los problemas y el pensamiento ordinario; la visión de sentido común: los conocimientos son presentados como ciertos, obvios, de sentido común, olvidando que la construcción científica, parte precisamente del cuestionamiento sistemático de lo obvio, etc.

Es muy probable que las creencias que los estudiantes tienen sobre la ciencia, los científicos y su trabajo tomen elementos de cada una las categorías de visión de ciencia mencionadas; más que caer en una de ellas, hay una combinación de elementos de las distintas visiones, en todo caso la limitación es que el estudio no puede ilustrar la clasificación en su totalidad. De ahí que sólo se ofrezcan elementos identificables con alguna de las visiones referidas.

Por lo que a las creencias evaluativo/recomendativas corresponde, se puede concluir que en general existe una tendencia, una disposición claramente favorable en su valoración sobre la ciencia, los científicos y su trabajo.

Una reflexión final que surge tanto de la práctica personal como de la observación no sistemática de la práctica docente de algunos profesores que enseñan ciencias y de la revisión de literatura hecha para este estudio, gira en torno a la necesidad de ir más allá en 
lo que tradicionalmente ha sido la enseñanza de la ciencia. Cada vez es mayor la conciencia de la importancia que en la educación integral del ser humano tiene la formación sistemática no sólo de conocimiento, sino de actitudes adecuadas, de acuerdo con el desarrollo del educando y los requerimientos de su incorporación a la sociedad en que ha de vivir. La consecuencia más importante de la desatención de esta dimensión, es el hecho de que aunque el alumno obtenga aparentemente resultados satisfactorios en cuanto a la calificación en los exámenes, referidos a los aspectos de conocimiento, al no haberse logrado la formación de una actitud correspondiente, al campo específico de la materia o a la adquisición del conocimiento en general, el alumno carece de una formación integral.

La actual psicología del aprendizaje señala que aparte del aprendizaje directo de los conceptos y habilidades de un determinado campo del saber, existe siempre un aprendizaje concomitante, que es una actitud favorable o desfavorable hacia el aprendizaje directo obtenido y que determina los efectos posteriores. En los currícula actuales hay una tendencia a incorporar en los programas escolares las actitudes que el maestro debe procurar cimentar en sus alumnos. El ocuparse de las actitudes como parte de las metas de los actuales programas escolares, corresponde a la comprensión a que se ha llegado en cuanto al papel fundamental que tienen éstas, como elementos esenciales de la personalidad y del comportamiento. Si la educación pretende, en definitiva, la formación valiosa de la personalidad y siendo la actitud, estructura básica de ésta, la problemática de formación, manifestación y cambio de actitudes es asunto importante del que deberá ocuparse la educación científica. 